\title{
Piezotronic and Piezo-phototronic Logic Computations Using Au Decorated ZnO Microwires
}

Haixia Li, ${ }^{a}$ Nishuang Liu, ${ }^{a, *}$ Xianghui Zhang, ${ }^{a, b}$ Jun Su, ${ }^{a}$ Luying Li, ${ }^{a}$ Yihua Gao ${ }^{a, b, *}$ and Zhong Lin Wang $^{c}$

${ }^{a}$ Center for Nanoscale Characterization \& Devices (CNCD), Wuhan National Laboratory for Optoelectronics (WNLO) and School of Physics, Huazhong University of Science and Technology (HUST), Luoyu Road 1037,Wuhan 430074, P.R. China

${ }^{\mathrm{b}}$ Hubei Collaborative Innovation Center for Advanced Organic Chemical Materials, Faculty of Physics \& Electronic Science, Hubei University, Wuhan 430062, P.R. China

${ }^{c}$ School of Materials Science and Engineering, Georgia Institute of Technology, Atlanta, Georgia 30332-0245, United States.

*Corresponding email: gaoyihua@ hust.edu.cn; nishuang_liu@foxmail.com

\begin{abstract}
Using the coupling effect of localized Schottky barriers formed by Au nanoparticles decorated $\mathrm{ZnO}$ microwires (MWs) and piezoelectric potential created upon straining, the highly enhanced piezotronic and piezo-phototronic effect have been applied for logic computations, i.e. designing strain-gated transistors (SGTs) and light-strain-gated transistors (LSGTs) to process mechanical and optical stimuli on the devices into electronic controlling signals. Linking four (L) SGTs following the connection rules, two series logic devices, such as inverter, NAND, AND, NOR, OR and XOR gates with good rectifying behaviors have been fabricated. Moreover, a 2:1 multiplexer can be designed for two independent input square wave signals with different frequencies. The light-strain-gated piezo-phototronic logic device based on Au NPs decorated ZnO MWs may find applications in active flexible electronics, optical micro/nanoelectromechanical systems, tunable bio-optoelectronic probes, and adaptive optical computation.
\end{abstract}


Keywords: localized Schottky barriers, piezoelectric potential, strain-gated transistors, lightstrain-gated transistors

\section{Introduction}

Recently, the coupling among piezoelectric potential, semiconductor properties, and optical excitation in wurtzite structured piezoelectric semiconductors, such as $\mathrm{ZnO}, \mathrm{CdS}$ and $\mathrm{GaN}$, has resulted in novel fundamental new phenomena, excellent device characteristics and various applications, leading to extensive attentions in the emerging field of piezophototronics.[1-6] Among the three materials, $\mathrm{ZnO}$ nanowire (NW) is of particular interest for investigating piezo-phototronic effect and implementing related applications due to its ultraviolet (UV) range optical response and intrinsic piezoelectric property. $\mathrm{ZnO}$ is an important material and its nanostructures has been widely studied for field effect transistors (FETs),[7] UV photodetectors,[8] light emitting diodes,[9] piezoelectric transducer,[10] and solar cells.[11] Especially, via coupling semiconducting and piezoelectric properties of $\mathrm{ZnO},[12]$ nanogenerator,[13, 14] piezoelectric FETs,[15] piezoelectric diodes,[16] and piezoelectric humidity/chemical sensors[17] were demonstrated.

Up to now, most of the piezoelectric effect is using polarization charges created at metal/ZnO electrode contact under strains to modulate transport of local charge carriers across the $\mathrm{SB}$, in which the strain stimuli applied on $\mathrm{ZnO} \mathrm{NW}$ are converted into electronic controlling signals. For example, the NW piezoelectric strain-gated transistors (SGT) was introduced[2, 18] based on a two-terminal metal-semiconductor-metal (M-S-M) structure, using the piezoelectric potential in the NW to modulate the charge carrier transport at the contacts without applying a gate voltage as in traditional FET. However, the sensitivity (control of strain on current) is still not good enough for piezoelectric sensor due to weak couplings among the characteristics of piezoelectricity, photoexcitation and semiconductor. For example, an on/off ratio of 112 was observed for piezoelectric gated transistor,[19] where the working current was restricted to a small range and may limit its practical application. 
Therefore, it must be necessary to develop an effective strategy to obtain high sensitivity by using strong couplings among the characteristics before the high performance principle devices of piezotronic and piezo-phototronic logic computations is realized. Our effective strategy is decorating Au nanoparticles (NPs) on $\mathrm{ZnO}$ surface to enhance the couplings and realizing the piezotronic and piezo-phototronic logic computations based on the enhanced coupling.

As stated above, piezoelectric potential and Schottky barrier (SB) at the $\mathrm{ZnO} / \mathrm{metal}$ electrode contact is used as a "gate" that dictates the electrical transport properties of the piezoelectric device. However, the control level is limited. According to previous report, in the case of metal particles fabricated on a semiconductor surface, the Fermi levels in the two materials must be coincident, which results in charge transfer and band bending near the interface in analogy with that on the interface between the metal and semiconductor. Thus the $\mathrm{Au}$ NPs can be treated as a lot of SBs on the surfaces of $\mathrm{ZnO},[20-22]$ which deplete more carriers near the $\mathrm{ZnO}$ surface, thicken the depletion region. In our previous study, we proved that the localized SBs (by Au NPs decoration) at the $\mathrm{ZnO}$ surface is much enhanced the sensitivity of the piezoelectric devices.[23] Therefore, we believe that the numberd SBs can be functioned as traditional SB at the electrode to improve performance of the device, and using the coupling effect of piezoelectric potential and localized SBs to fabricate piezoelectric two-terminal transistors must be important to enhance the current on/off ratio of the devices. Furthermore, the piezo-phototronic effect has been applied to design two-terminal transistors in our study, which process mechanical and optical stimuli on the devices into electronic controlling signals.

In this study, $\mathrm{ZnO}$ microwire (MW) (L)SGTs were fabricated by using the piezoelectric polarization charges created at the metal/ZnO interface under strains and UV light to modulate transport of local charge carriers across the SB. Linking four (L)SGTs following the connection rules, two series logic devices such as inverter, NAND, AND, NOR, OR, XOR 
and 2:1 multiplexer (MUX) gates with good rectifying behaviors have been fabricated. Here, we got a high $\mathrm{I}_{\max } / \mathrm{I}_{\min }$ current ratio $9.3 \times 10^{4}$, which is much higher than the former ratios in this field. The light strain gated piezo-phototronic logic devices based on Au NPs decorated $\mathrm{ZnO}$ MWs may find applications in active flexible electronics, optical micro/nanoelectromechanical systems, tunable bio-optoelectronic probes, and adaptive optical computing.

\section{Experimental Section}

$\mathrm{ZnO}$ MWs were obtained by one step chemical vapor deposition and discrete Au NPs were formed on $\mathrm{ZnO}$ surface by spraying deposition.[23] The Au NPs distribution on the upside of the surface (the bottom side surface is almost smooth) due to $\mathrm{ZnO} \mathrm{MW}$ is lying down. The asobtained $\mathrm{ZnO} \mathrm{MWs}$ have lengths of several hundred micrometers, diameters of $0.5 \sim 10 \mu \mathrm{m}$. The piezoelectric SGTs were fabricated by transferring and bonding single $\mathrm{ZnO} \mathrm{MW}$ with and without Au NPs laterally on the polyimide substrate (1 mm thick), respectively. Two ends of a MW fixed by silver paste served as source and drain electrodes, respectively, and an M-S-M structure device was formed. A thin layer of polydimethylsiloxane was used to package the device, which not only enhanced the mechanical robustness of the whole device but also prevented the degradation of the $\mathrm{ZnO} \mathrm{MW}$ by gas or moisture in the environment.

The morphology and crystalline structure of the as-deposited $\mathrm{ZnO}$ MWs were characterized by a SEM (FEI Nova Nano-SEM 450) and TEM (FEI Titan G2 60-300). UV photo-response measurement was carried out between two electrodes at a fixed bias voltage of $8 \mathrm{~V}$ by switching the light from a portable UV lamp $\left(\lambda=365 \mathrm{~nm}, 0.3 \mathrm{~mW} \mathrm{~cm}{ }^{-2}\right)$ "on" and "off".

\section{Results and Discussion}

Figures 1a-c show the working schematics of a traditional Junction field-effect-transistor (JFET). For an n-channel JFET, two P-type doped regions are used as grid electrode and both 
ends of n-type semiconductor are used as drain and source; the current flowing from the drain to source under an applied external voltage $\mathrm{U}_{\mathrm{DS}}$ is controlled by the gate voltage $\mathrm{U}_{\mathrm{GS}}$, resulting in the change of the channel width for transporting the carriers.

Analogously, Figure 1d shows the schematics of the piezoelectric transistor with metal$\mathrm{ZnO}$-metal structure, which formed $\mathrm{SB}$ at the electrode interface. When applying a strain, the created piezoelectric charges at the interface region control the carrier transport at the metal/ZnO interface, which forms a piezoelectric transistor. Thus, the externally applied gate voltage $\mathrm{U}_{\mathrm{GS}}$ in a traditional JFET is replaced by an inner crystal potential in this piezoelectric transistor, which means that the piezoelectric transistor only needs external drain and source terminal. The controlling channel width in a traditional JFET is replaced by controlling the interface via inner crystal potential, and the current transported across the interface is the exponential of the local barrier height in the reversely biased case, the on/off ratio can be rather high due to the nonlinear effect. Thus, a voltage controlled device is replaced by a strain-controlled device via varying the local barrier height.[24] In Figure 1d and e, the external stimulus can be strain/light, which can increase/decrease the depletion region.

Figure 1e is the schematic of the Au NPs decorated piezoelectric transistor with metalZnO-metal structure. As we stated above, the Au NPs are treated as a lot of SBs on the surfaces of $\mathrm{ZnO}$, which can improve performance of the device. Our previous studies proved that the coupling between piezoelectric potential and numberd SBs (by Au NPs decoration) at the $\mathrm{ZnO}$ surface can much enhance the sensitivity of the piezoelectric devices. ${ }^{[23]}$ For the $\mathrm{Au}$ NPs decorated piezoelectric transistor, the electrical transport properties of the device will be controlled more effectively by both the SB at the electrode contact and numberd SBs on the surface. The coupling among the characteristics of piezoelectricity, photoexcitation and semiconductor are enhanced because the numbered decorated Au NPs on the $\mathrm{ZnO}$ surface play the role of SBs, which supplies new and important control for field-effect-transistor (FET). Moreover, the input of the external stimulus can be also light which change the SB 
height and the carriers' density. Figure 1f show the schematics of the logic units corresponding to our work, there are two kinds of stimulus (UV light and strain) corresponding to the (L)SGTs.

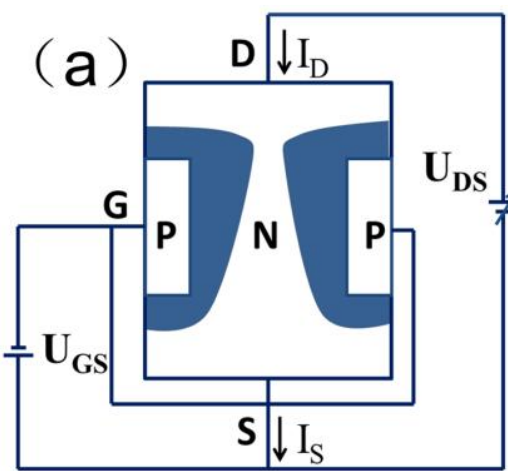

(d)

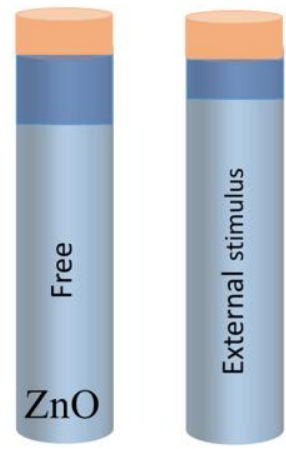

Depletion region

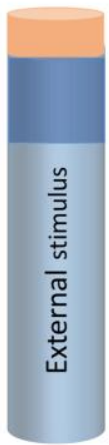

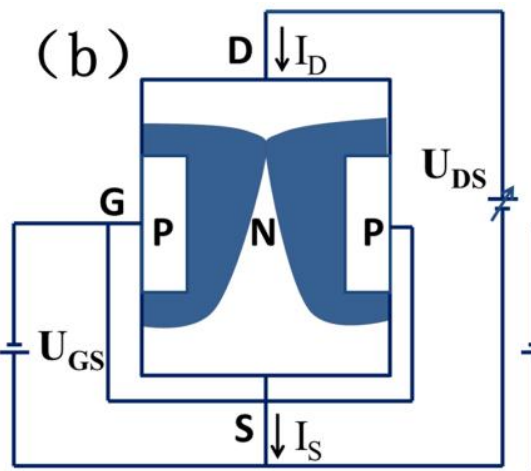

(e)
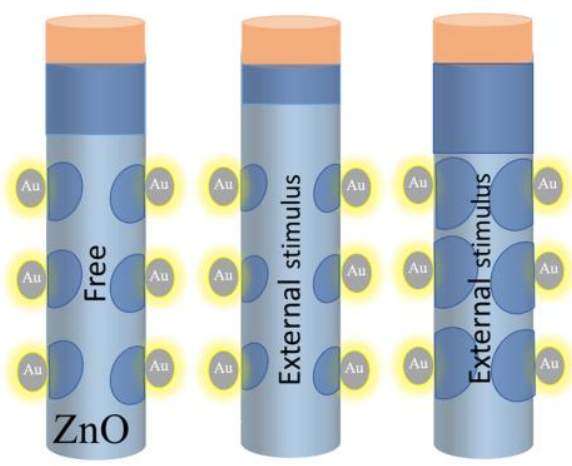

Schottky contact

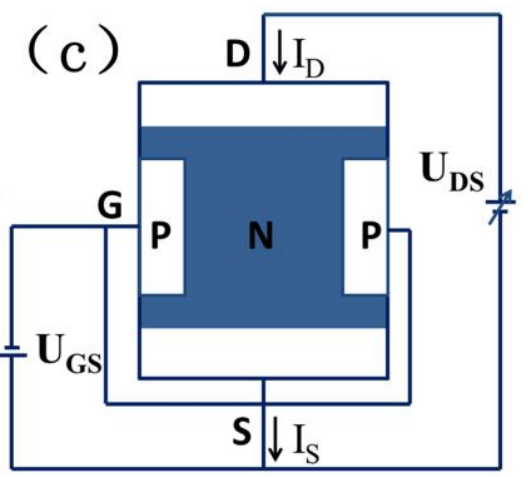

(f)

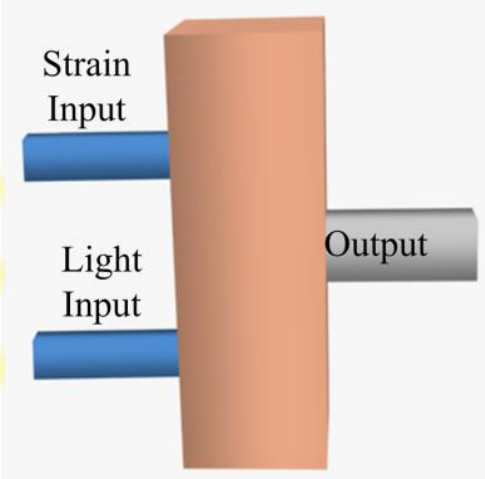

Au nanoparticles

Figure 1. The working mechanism of the devices. (a), (b), (c) Schematics of a traditional JFET. (d) Schematics of a piezoelectric transistor with metal-ZnO-metal structure. (e) Schematics of the Au NPs decorated piezoelectric transistor with metal-ZnO-metal structure. In (d) and (e), the external stimulus can be strain/light, which can increase/decrease the depletion region. Then piezotronic and piezo-phototronic logic computations were realized, as shown in the schematic of the logic unit (L) SGTs in (f).

The single crystallinity of the $\mathrm{ZnO}$ MWs has been confirmed, and more details of the interface area between Au NPs and ZnO MW were shown by a high resolution transmission electron microscopy (HRTEM) image in Figure 2a. There are two distinct sets of lattice fringes at the interface, one of the lattice spacing of $0.52 \mathrm{~nm}$ is attributed to the [0001] planes 
of the hexagonal wurtzite $\mathrm{ZnO}$ crystal[25] and the other of the $0.235 \mathrm{~nm}$ is to the $\mathrm{Au}(111)$ planes.[26] As shown in typical SEM image of Figure 2b, an M-S-M structure device was formed, two ends of a Au NPs decorated ZnO MW fixed by silver paste served as source and drain electrodes, respectively. The Au NPs are uniformly and discretely distributed on the surface of $\mathrm{ZnO}$ wire, as shown in the right part of the Figure $2 \mathrm{~b}$. Figure $2 \mathrm{c}$ is a picture of a flexible SGT which can be implemented periodic compressive and tensile strain.
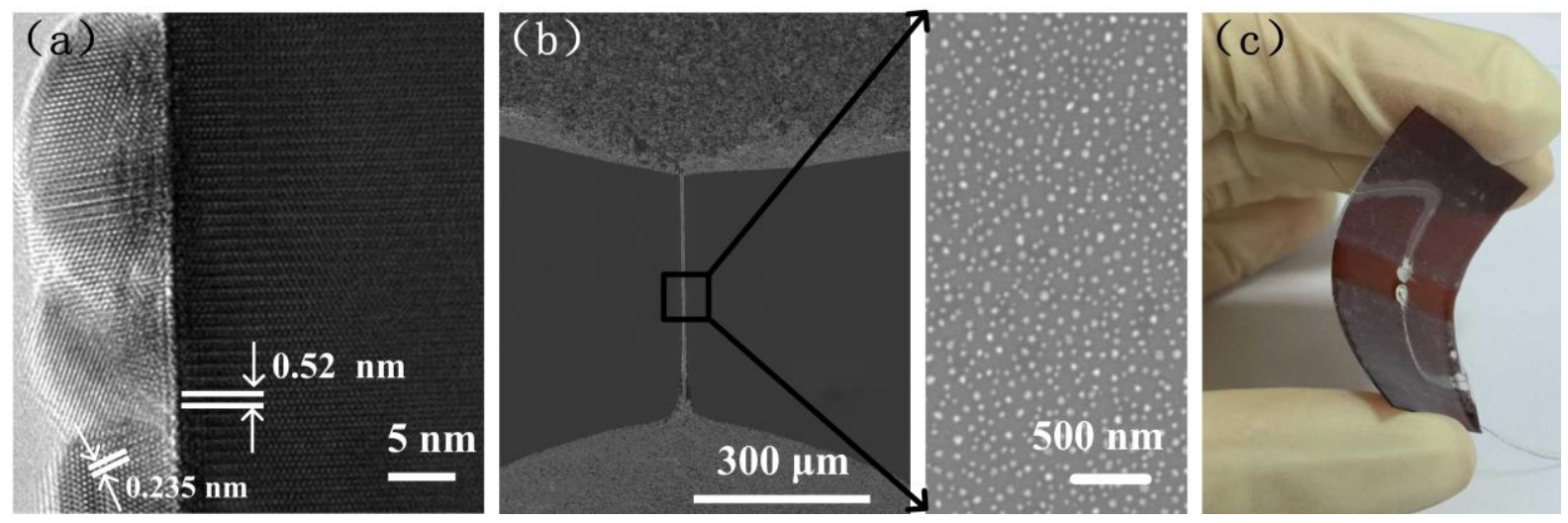

Figure 2. The microstructure of the Au NPs decorated ZnO MW. (a) A high resolution TEM image of the Au NPs decorated ZnO MW. (b) SEM images of an Au NPs decorated ZnO MW based flexible SGT. The right part of (b) show more details of the uniform distribution of $\mathrm{Au}$ NPs on the surface of $\mathrm{ZnO}$ wire. (c) The picture of a flexible SGT on which strain can be implemented.

Once the substrate was bent, a compressive/tensile strain was created in the MW because the mechanical behavior of the entire structure was determined by the substrate. The magnitude and the sign of applied strains can be calculated following the method reported elsewhere.[27] Figures 3a (without Au NPs) and 3b-c (with Au NPs: b is tensile strain and c is the compressive strain) show the device's drain-source current in the dark condition as a function of voltage under various applied strain (the tensile/compressive strains are defined as positive/negative, respectively). Generally, the nonlinear behavior shown in figure $3 \mathrm{a}, \mathrm{b}$ and c should be related to the SB formed between the $\mathrm{ZnO}$ and the silver paste electrodes,[28, 29] 
and the shape of the I-V curve depends on the heights of the SB height, which functions as the controlling input signal to gate the electrical transport in the SGT.

It is obvious that the dark current experienced a weak decrease from the state of tensile strain to the state of compressive strain. The reason for the decreased dark current of the original $\mathrm{ZnO}$ device was analyzed as following. When a SGT is under strain, two typical effects may affect the carrier transport process. One is the piezoresistance effect because of the change in bandgap, charge carrier density, and possibly the density of states in the conduction band of the semiconductor crystal under strain. This effect is a symmetric effect on the two ends of the contacts without polarity and producing the function of a device.[1] The other reason for the decreased dark current of the original device was analyzed by piezoelectric effect: when compressive strain is introduced, both the band structure and piezoelectric potential would contribute to the rise in the SB height at the electrode contact, resulting in the further upward bent of conduction $\left(E_{c}\right)$ and valence $\left(E_{v}\right)$ bands at the surface of the $\mathrm{ZnO} \mathrm{MW}$.

For the device with Au NPs decoration (Figures 3b and c), the dark currents in figure $3 a$ (without $\mathrm{Au}$ ) is $4.58 \times 10^{-4} \mathrm{~A}$ and that in figure $3 \mathrm{~b}$ (with $\mathrm{Au}$ ) is $1.04 \times 10^{-4} \mathrm{~A}$ at the fixed bias of $20 \mathrm{~V}$ under the equivalent strain-free states. After covering with Au NPs, the negatively charged $\mathrm{Au}$ NPs depletes the carriers near the surface of $\mathrm{ZnO} \mathrm{NW}$, and numbered SBs are formed at the $\mathrm{Au}$ and $\mathrm{ZnO}$ interface.[23] Since the width of depletion layer is related to barrier height, depletion region near the Au NPs is larger than the depletion region far from the Au NPs. The formation of the large depletion region is the main reason for the decrease in the dark current after covering with Au NPs. The above working principle of the Au NPs decorated $\mathrm{ZnO}$ surface can be explained using band diagrams shown in supplementary information Figure S1. Once the substrate was bent, it is obvious that the dark current experienced a drastically decrease from the state of tensile strain to the state of compressive strain. The reason for the more decreased dark current is explained by using Au NPs 
introduced SBs as stated above, after introducing strain, the SB height is increased much higher under equivalent compressive strain due to more SBs induced by Au NPs, and thereby the current has a sharp decrease as compared with the original ZnO MW as we described in Figure 3a. However, when a tensile strain is introduced, the SGT's band structure changes and opposite polarity piezoelectric potential generates, $[6,19]$ and at last the depletion region width and SB height decrease. Thus, the I-V curves shift upward with tensile strain and downward with compressive strain. The above our experiments prove that the localized SBs at the $\mathrm{ZnO}$ surfaces can help improve the piezo-performance.

As shown in Figure 3d, the photocurrent of the Au NPs decorated ZnO MW SGT also increases as the applied tensile strain increases and reduces as the applied compressive strain increases. The increase of photocurrent is explained as follows, when the device is illuminated by UV light, the stronger and expanding built in field enhances the spatial separation effect, and increases the photo-response gain.[30] The experiments demonstrated that optical illumination with appropriate energy and intensity can therefore modulate the conductivity of the $\mathrm{ZnO} \mathrm{MW}$ device and turn it into electrically "on" state.

Furthermore, as shown in Figure 3e, the Au NPs decoration SGT was measured under various applied strain for several cycles. The current descend/rise by almost the same value in each cycle of straining and fully recover, being very similar to other literatures.[18, 19] For the $\mathrm{ZnO}$ MW SGT, the external strain $\left(\varepsilon_{\mathrm{g}}\right)$ acts as the gate input for controlling the on/off state of the SGT. Figure 3f, the drain/source current strain $\left(\mathrm{I}_{\mathrm{DS}}-\varepsilon_{\mathrm{g}}\right)$ curves of the SGT under different bias voltage show that the current increases as the applied tensile strain increases and reduces as the applied compressive strain increases, where the threshold gate strain $\varepsilon_{\mathrm{T}}$ is around $-0.368 \%$. The on and off state for the SGT can be determined as the values obtained at $\varepsilon_{\mathrm{g}}(\mathrm{off})=\varepsilon_{\mathrm{T}}-0.3 \%$ and $\varepsilon_{\mathrm{g}}(\mathrm{on})=\varepsilon_{\mathrm{T}}+0.7 \% . \mathrm{I}_{\max }($ strain of $0.98 \%)=0.3 \mathrm{~mA}$ and $\mathrm{I}_{\min }=3.23 \mathrm{nA}$ (strain of $-0.81 \%$ ) are hence obtained with the $\mathrm{I}_{\max } / \mathrm{I}_{\min }$ ratio of $9.3 \times 10^{4}$ at about $5 \mathrm{~V}$ fixed bias, which is much higher than the research. [19] 

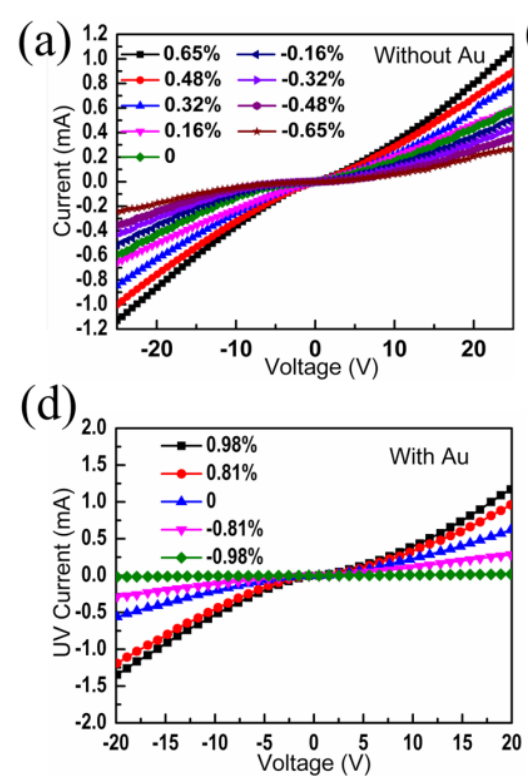

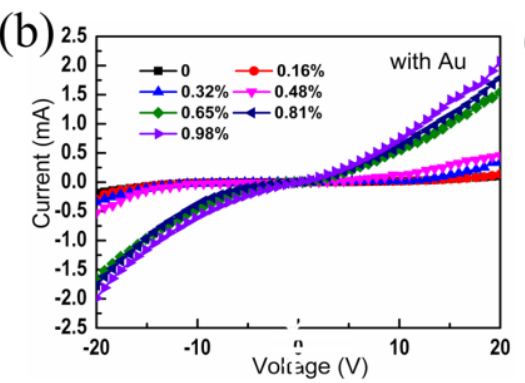

(c)

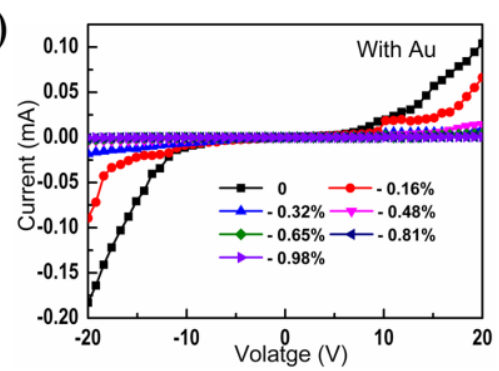

(e)
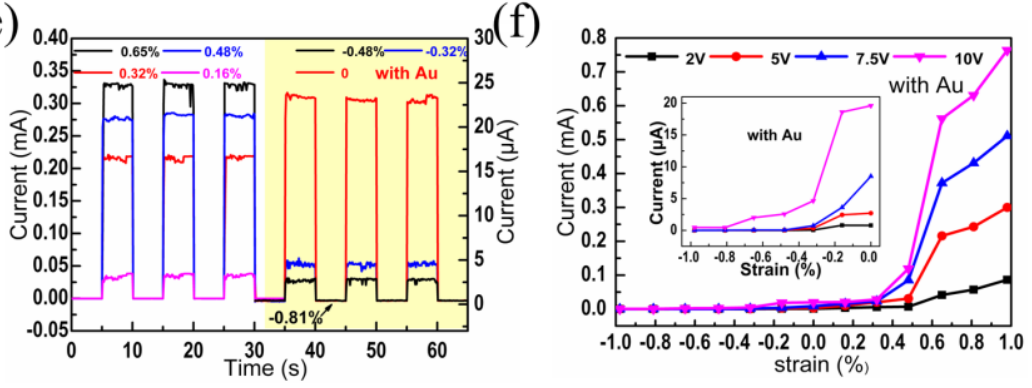

Figure 3. The I-V, I-T characteristics and of various devices. (a), (b) and (c), Typical I-V characteristics of the SGT at different strains without (a) and with (b, c) Au NPs. (b) is in tensile strain and (c) is in compressive strain. (d) Photocurrent of the Au NPs decorated $\mathrm{ZnO}$ MW SGT. (e) Current response of the Au NPs decorated SGT under alternative compressed/tensile strain at a fixed bias of $7.5 \mathrm{~V}$. (f) $\mathrm{I}_{\mathrm{DS}^{-} \varepsilon_{\mathrm{g}}}$ curves of the typical $\mathrm{ZnO} \mathrm{MW}$ SGT measured at different fixed bias about $2 \mathrm{~V}, 5 \mathrm{~V}, 7.5 \mathrm{~V}$ and $10 \mathrm{~V}$, the inset showing the detail of the compressive strain.

Two series of experiments were carried out to investigate the coupling of local SBs and piezoelectric effects on the performances of the piezoelectric strain gated inverters (SGIs) with and without Au NPs. We fabricated an inverter with two SGTs both on the top and bottom surfaces of the substrate, as shown in Figures 4a and c. The two SGTs have absolutely contrary on/off state, depending on whether the substrate is bent upward or downward. For the SGI logic unit, the strain input logic "1" is defined as: the upside \#1 SGT is "off"compressive and the downside \#2 SGT is "on"-tensile, leading to the electrical output logic " 0 "-a lower voltage, while the strain input logic " 0 " is defined as: the upside \#1 SGT is "on"tensile and the downside \#2 SGT is "off"-compressive, leading to the electrical output logic "1"-a higher voltage. These definitions are adopted in all demonstrations here and afterwards. 

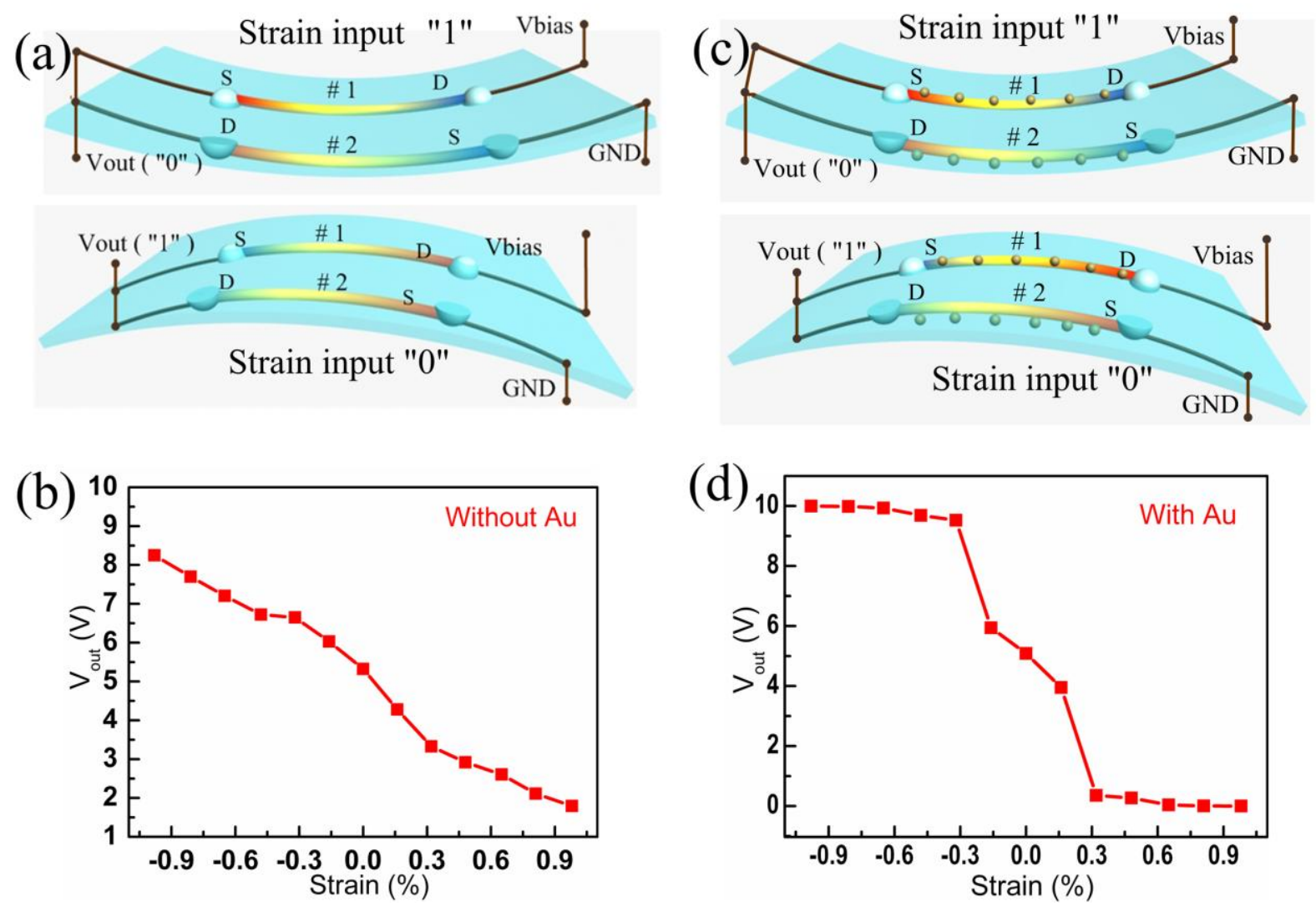

Figure 4. Schematics of two ZnO MW piezoelectric SGIs without (a) and with (c) Au NPs decoration performing logic operations in response to the strain inputs. Experimental output voltages with the strains of the ZnO MW piezoelectric SGIs without (b) and with (d) Au NPs.

The strain voltage transfer characteristic of the two SGIs were obtained by plotting the output voltages versus the applied external strains, as shown in Figures $4 \mathrm{~b}$ and d. $\mathrm{V}_{\mathrm{OH}}$ and $\mathrm{V}_{\mathrm{OL}}$ represent the high and low output voltage of the SGI, with theoretical values of $\mathrm{V}_{\mathrm{OH}}=$ $\mathrm{V}_{\text {bias }}=10 \mathrm{~V}$ and $\mathrm{V}_{\mathrm{OL}}=0 \mathrm{~V}$. Here, the logic swing of the two piezoelectric inverters are defined as $\mathrm{V}_{\mathrm{OH}}-\mathrm{V}_{\mathrm{OL}}=8.25 \mathrm{~V}-1.79 \mathrm{~V}=6.46 \mathrm{~V}$ (without $\mathrm{Au}$ ) and $9.99 \mathrm{~V}-0.002 \mathrm{~V}=9.988 \mathrm{~V}$ (with Au NPs) according to the experimental values, respectively. The difference between experimental and theoretical values was probably caused by the voltage drop across the SGT which was "on" during the measurement for the two SGIs. Figure $4 \mathrm{~b}$ shows that the original ZnO MW based SGI has a lower $\mathrm{V}_{\mathrm{OH}}$ and a higher $\mathrm{V}_{\mathrm{OL}}$, and the output voltages were restricted to a small range, which blocks its practical application. For the Au NPs decorated SGI with a higher $\mathrm{V}_{\mathrm{OH}}$ and a lower $\mathrm{V}_{\mathrm{OL}}$, when the applied strain is in the region of $\varepsilon_{\mathrm{g}}<-$ 
$0.48 \%$, the electrical output is logic " 1 "; when the applied strain is in the region of $\varepsilon_{\mathrm{g}}>0.48 \%$, the electrical output is logic " 0 ". It is obvious that the Au NPs decorated SGI is easier to implement logic transformation than the original $\mathrm{ZnO}$ SGI. The difference between the SGIs with and without Au NPs decoration were caused by controlling the electric transport process of the $\mathrm{ZnO}$ more effectively due to localized SBs introduced.
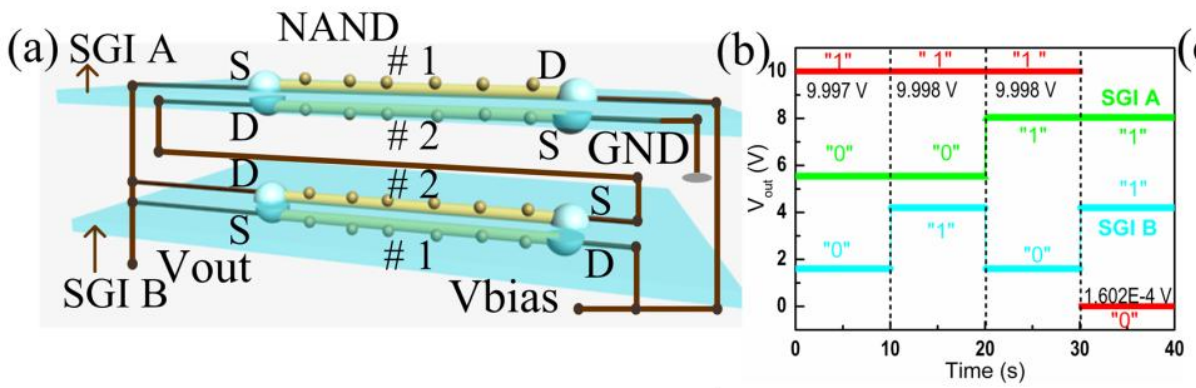

(d)

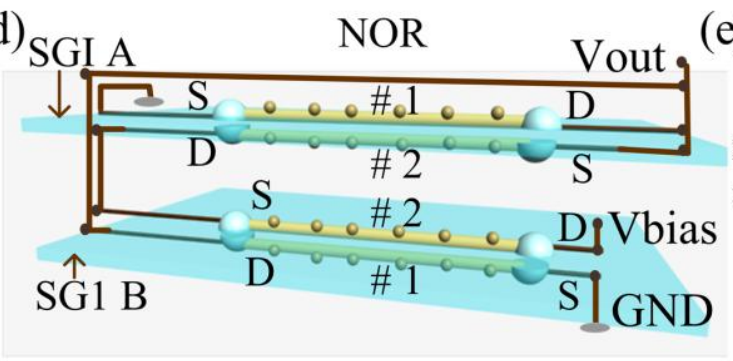

(e)

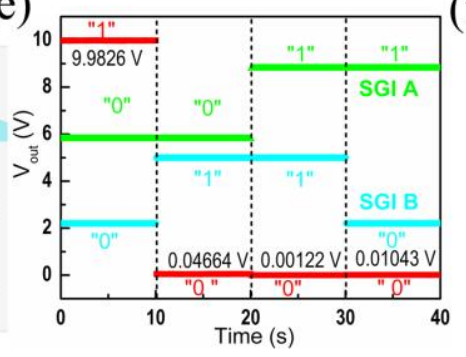

(c)

\begin{tabular}{cc}
\hline$\varepsilon_{\mathrm{G}}$ & $\mathrm{V}_{\text {out }}$ \\
\hline$" 00 "$ & "1" \\
\hline $01 "$ & $" 1 "$ \\
\hline $10 "$ & $" 1 "$ \\
\hline $111 "$ & $" 0 "$
\end{tabular}

(f)

\begin{tabular}{|c|c|}
\hline$\varepsilon_{G}$ & $V_{\text {out }}$ \\
\hline "0 0 " & "1" \\
\hline "0 1" & "0" \\
\hline "1 1" & "0" \\
\hline "1 0" & "0" \\
\hline
\end{tabular}

Figure 5. Strain controlled piezoelectric logic gates and some basic operations based on Au NPs decorated ZnO MWs. (a), (b) and (c): piezoelectric logic NAND gate. (d), (e) and (f): piezoelectric logic NOR gate. (a), (d): Schematic of the Au NPs decorated ZnO MW piezoelectric NAND and NOR gate. (b), (e) Shows the measured electrical output voltages of NAND and NOR gate versus the input strains (time). (c), (f) Experimental truth table of the output voltage with the two SGIs at various strain inputs.

More universal piezoelectric logic operations were obtained by systematically integrating the Au NPs decorated ZnO MW SGIs. According to corresponding connection rules (Figures $5 \mathrm{a}, \mathrm{d})$, the measured output voltages of the NAND and NOR gate are plotted in Figures 5b, e. Red lines are the electrical output voltages of logic gate; blue and green lines represent the strain input applied on SGIs A and B, respectively, where strain input " 0 " and " 1 " are defined 
in Figure 4c. The output and input signals are listed in the truth table in Figures $5 \mathrm{c}, \mathrm{f}$. In a similar manner, piezoelectric strain controlling AND and OR gate have also been realized by integrating SGIs, as presented in Figure S2.

Strain-electricity coupling can also be used to design logic gates. Figure 6 shows an XOR gate and 2:1 MUX. For the XOR (Figure 6a), the drain electrode of \#1 SGT is connected to the electrical input Vo while the drain electrode of \#2 SGT is connected to Vō, which is the logically compensate electrical input to Vo. The strain gated input logic for \#2 SGT and \#1 SGT also compensate each other. The change in the connections of the electrodes from those demonstrated in the SGI results in different logic functions. When the substrate is bent upward or downward, the electric output would be either "1" or "0". For the XOR gate shown in Figure 6a, in the upside state, the electrical output is logic "0" when the strain input is logic "1" and the electrical input Vo applied on \#1 SGT is logic "1"; while in the downside state, the electrical output is logic " 1 " when the strain input is logic " 0 " and the electrical input Vo applied on \#1 SGT is logic "1". As a supplementary description without the schematics here, the electrical output is logic " 1 " when the strain input is logic " 1 " and the electrical input Vo applied on \#1 SGT is logic "0"; while the electrical output is logic "0" when strain input is logic " 0 " and the electrical input Vo applied on \#1 SGT is logic "0". All four output voltages of the XOR gate versus the input gate strains and electrical input are shown in Figure 6b.

The 2:1 MUX was realized according to corresponding connection rules in Figure 6c, where the drain electrodes of \#1 SGT and \#2 SGT are connected independently to two square wave signals with different frequency (input 1 with $0.5 \mathrm{~Hz}$ and input 2 with $1 \mathrm{~Hz}$ ) rather than logically complements. For the upside state of Figure 6c, \#1 SGT is "off” and \#2 SGT is “on", hence the electrical output is determined by \#2 SGT and obtained frequency is $1 \mathrm{~Hz}$. For the downside state of Figure 6c, \#1 SGT is "on" and \#2 SGT is "off", hence the electrical output is determined by \#1 SGT and the obtained frequency is $0.5 \mathrm{~Hz}$. The measured electrical 
output voltages and input signals with the two states are listed in the Figure 6d. Analogously, by expanding the 2:1 MUX, an n:1 MUX based on the same mechanism as above can be obtained, which enables us to pick one of the $\mathrm{n}$ inputs and direct it to the output.
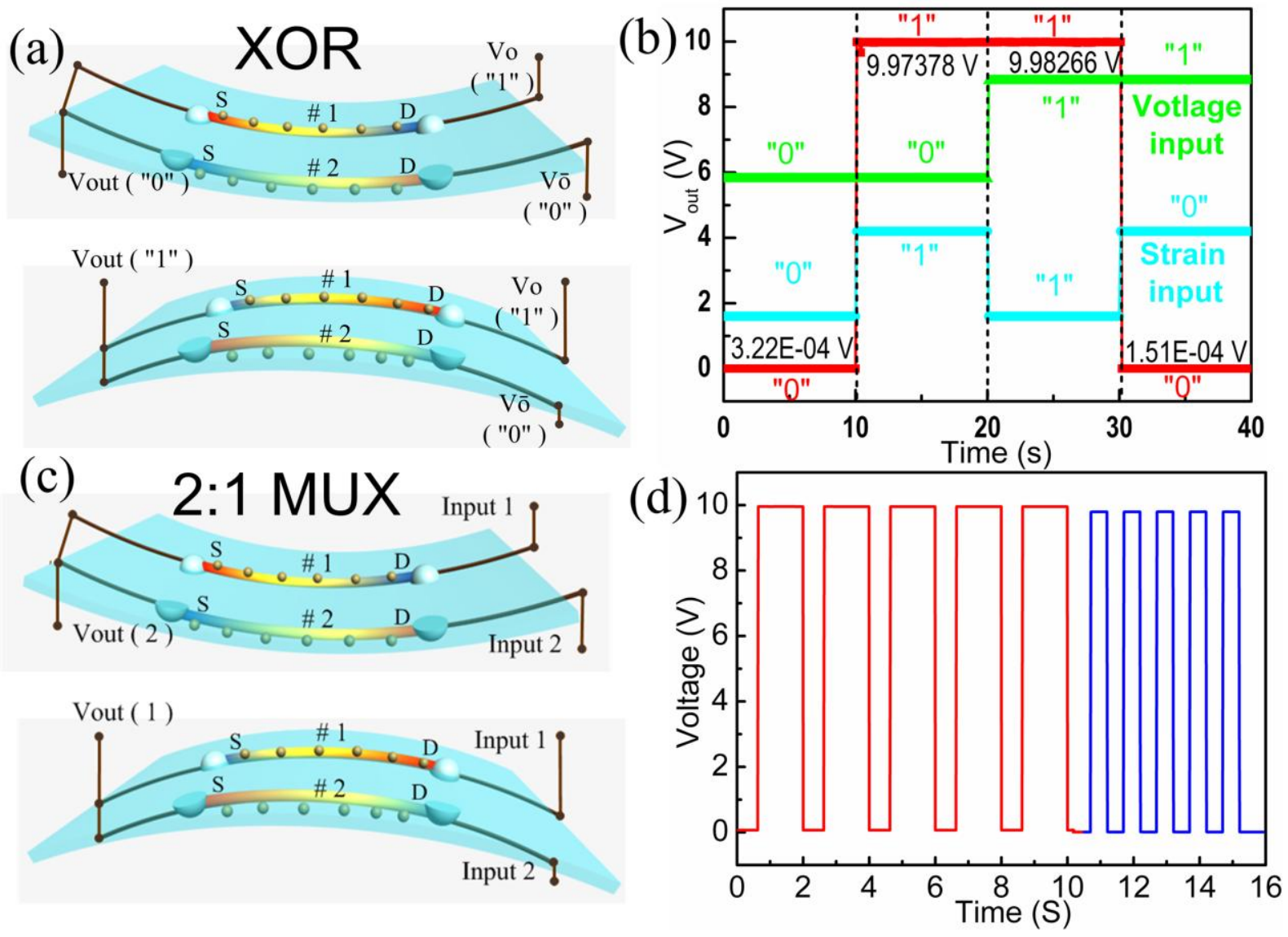

Figure 6. Different from the above gates in Figures 4, 5 based on strain coupling, the gates are based on strain-electricity coupling. (a) and (b): Piezoelectric logic XOR gate. (c) and (d): 2:1 MUX. (a) Schematics of an Au NPs decorated ZnO MW XOR logic gate performing logic operations on strain and electrical inputs. (b) Logic operations and experimental truth table of the $\mathrm{ZnO}$ MW strain gated XOR logic gate. Red line is the electrical output of the XOR gate. Blue and green lines represent the electrical and strain inputs applied on \#1 SGT and \#2 SGT, respectively. (c) Schematics of an Au NPs decorated ZnO MW 2:1 MUX performing logic operations on two square wave signals with different frequency inputs. (d) The measured electrical output voltages in the two states. 
We have further studied the piezo-phototronic effect in two terminal Au NPs decorated ZnO MW SGT by introducing both light and strain to the device. The "dual gate" programmable composed of four LSGTs. Each of these four LSGTs has two independently configurable gate controlling signals $\mathrm{L}_{\text {in }}$ and $\varepsilon_{\text {in }}$ for light and strain inputs, respectively. The output from these logic units is electrical voltage. In the demonstration, the logic " 0 " state for $\mathrm{L}_{\mathrm{in}}$ is defined as focusing the $365 \mathrm{~nm} \mathrm{UV}$ laser with intensity of $0.3 \mathrm{~mW} \mathrm{~cm}{ }^{-2}$ on \#2 and \#4 LSGTs; the logic "1" state is defined as focusing the UV laser on \#1 and \#3 LSGTs. The logic “ 0 ” state for $\varepsilon_{\text {in }}$ is defined as applying $-0.98 \%$ compressive strain on \#1 and \#2 LSGTs; the logic "1" state is defined as applying $-0.98 \%$ compressive strains on \#3 and \#4 LSGTs. Figures $7 \mathrm{a}, \mathrm{d}$ and $\mathrm{g}$ illustrate the configurations of piezo-phototronic AND, OR, and XOR logic gates comprising of four LSGTs, where both $\mathrm{L}_{\text {in }}$ and $\varepsilon_{\text {in }}$ are logic “ 0 ”.

These piezo-phototronic logic operations are experimentally verified as shown in Figures $7 \mathrm{~b}$, e and h, with low voltage output (logic "0" output) at around $0 \mathrm{~V}$ and high voltage output (logic " 1 " output) at around $10 \mathrm{~V}$, which is the bias voltage $\mathrm{V}_{\text {bias. }}$ Red lines are the experimentally measured electrical output voltages of the logic gates, while green and blue lines represent the logic levels of input strains and UV laser, respectively. The truth table of the input and output signals are presented in Figures 7c, f and i. Other piezo-phototronic universal logic units, including NAND and NOR logic gates, were also successfully demonstrated (Figure S3). 


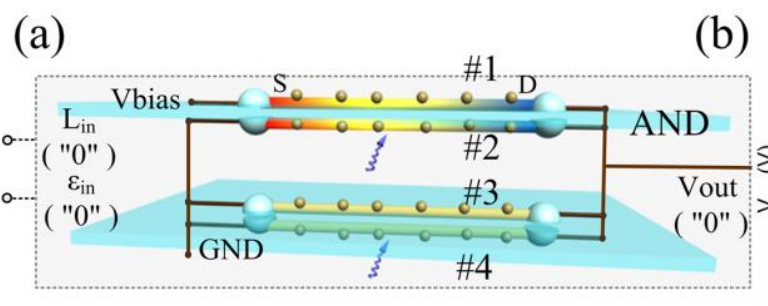

(b)
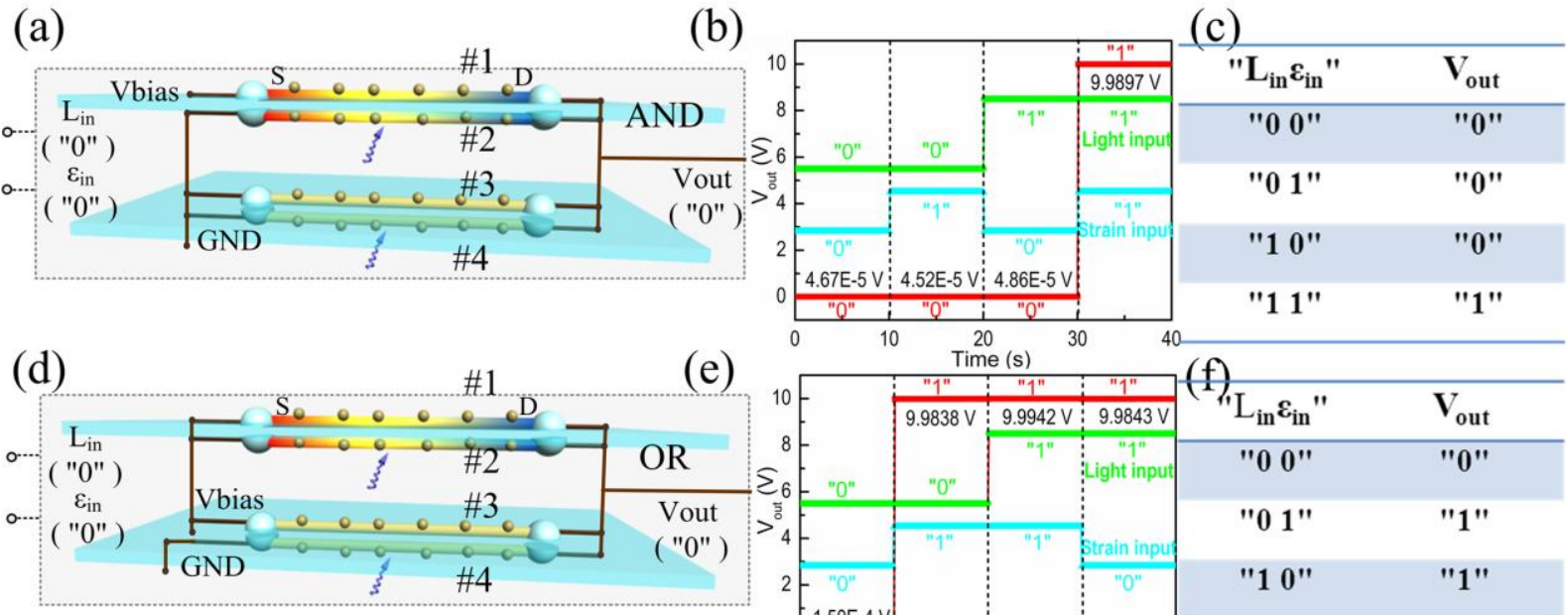

(e)
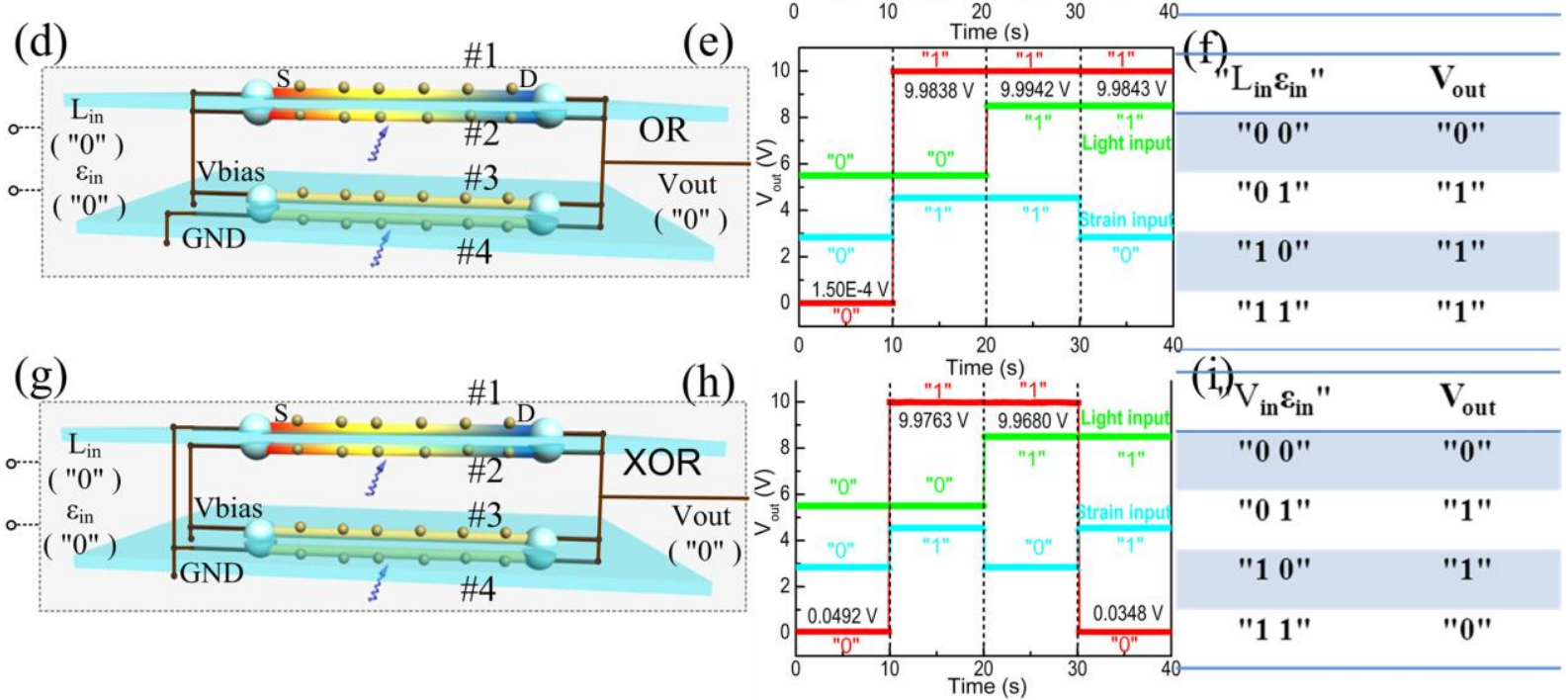

Figure 7. Different from the above gates in Figures 4-6, the gates are based on strain-light coupling, named piezo-phototronic logic computations. (a), (b) and (c) Piezo-phototronic logic AND gate. (d), (e) and (f) Piezo-phototronic logic OR gate. (g), (h) and (i) Piezophototronic logic XOR gate. (a), (d), (g) Schematic configurations of a piezo-phototronic logic AND, OR, XOR gates comprising of four LSGTs, taking optical and strain inputs combination $(0,0)$ as a demonstration with piezo-potential distribution in the MWs highlighted using color code. (b), (e), (h) Electric output signals of an AND, OR, XOR gates under four input states as labeled, $\mathrm{V}_{\mathrm{DD}}=10 \mathrm{~V}$. (c), (f), (i) Experimental truth table of AND, OR and XOR gates.

\section{Conclusions}

In summary, by using numberd $\mathrm{SBs}$ formed at $\mathrm{ZnO}$ surface, the highly enhanced piezotronic and piezo-phototronic couplings have been applied for logic computations to design SGTs and LSGTs, which process mechanical and optical stimuli on the devices into electronic controlling signals. Two series logic devices such as inverter, AND, OR, NAND, NOR, XOR 
gates with good rectifying behaviors and 2:1 MUX have been fabricated. The piezophototronic logic devices based on Au NPs decorated ZnO MWs may find applications in active flexible electronics, prosthetics, optical micro/nanoelectromechanical systems, tunable bio-optoelectronic probes, and adaptive optical computing.

\section{Acknowledgements}

This work was supported by the National Natural Science Foundation of China (11204093, 11374110, 51371085), Overseas Master Program (MS2011HZKJ043), and 'the Fundamental Research Funds for the Central Universities', HUST: 2014TS124, and U.S. Department of

Energy, Office of Basic Energy Sciences (Award DE-FG02-07ER46394). The authors would like to thank Prof. Wenzhuo Wu of Purdue University for the helpful discussion.

\section{References}

[1] Z.L. Wang, Nano Today, 5 (2010) 540-552.

[2] W. Wu, C. Pan, Y. Zhang, X. Wen, Z. L. Wang, Nano Today, 8 (2013) 619-642.

[3] Y. Hu, Y. Zhang, Y. Chang, R.L. Snyder, Z.L. Wang, Acs Nano, 4 (2010) 4220-4224.

[4] Q. Yang, W. Wang, S. Xu , Z. L. Wang, Nano Lett. 11 (2011) 4012-4017.

[5] C. Pan, L. Dong, G. Zhu, S. Niu, R.Yu, Q.Yang, Y. Liu, Z. L. Wang, Nat. Photonics 7 (2013) 752-758.

[6] R. Yu, W. Wu, C. Pan, Z. Wang, Y. Ding, Z. L. Wang, Adv. Mater. 27 (2015) 940-947.

[7] M. S. Arnold, P. Avouris, Z. W. Pan, Z. L.Wang, J. Phys. Chem. B 107 (2003) 659-663.

[8] C. Soci, A. Zhang, B. Xiang, S. A. Dayeh, D. P. R. Aplin, J. Park, X. Y. Bao, Y. H. Lo, D. Wang, Nano Lett. 7 (2007) 1003-1009.

[9] W. I. Park, G. C. Yi, Adv. Mater. 16 (2004) 87-+.

[10] B. A. Buchine, W. L. Hughes, F. L. Degertekin, Z. L. Wang, Nano Lett. 6 (2006) $1155-$ 1159.

[11] M. Law, L. E. Greene, J. C. Johnson, R. Saykally, P. Yang, Nat. Mater. 4 (2005) 455-459.

[12] Z. L. Wang, J. Phys. Chem. Lett. 1 (2010) 1388-1393. 
[13] Z. L. Wang, J. H. Song, Science 312 (2006) 242-246.

[14] X. Wang, J. Song, J. Liu, Z. L. Wang, Science 316 (2007) 102-105.

[15] X. Wang, J. Zhou, J. Song, J. Liu, N. Xu, Z. L. Wang, Nano Lett. 6 (2006) 2768-2772.

[16] J. H. He, C. L. Hsin, J. Liu, L. J. Chen, Z. L. Wang, Adv. Mater. 19 (2007) 781-784.

[17] C. S. Lao, Q. Kuang, Z. L. Wang, M.-C. Park, Y. Deng, Appl. Phys. Lett. 90 (2007) 262107.

[18] J. Zhou, P. Fei, Y. Gu, W. Mai, Y. Gao, R. Yang, G. Bao, Z. L. Wang, Nano Lett. 8 (2008) 3973-3977.

[19] W. Wu, Y. Wei, Z. L. Wang, Adv. Mater. 22 (2010) 4711-4715.

[20] B. J. Coppa, R. F. Davis, R. J. Nemanich, Appl. Phys. Lett. 82 (2003) 400.

[21] V. Dobrokhotov, D. N. Mcilroy, M. G. Norton, A. Abuzir, W. J. Yeh, I. Stevenson, R. Pouy, J. Bochenek, M. Cartwright, L. Wang, J. Dawson, M. Beaux, C. Berven, J. Appl. Phys. 99 (2006) 104302.

[22] V. P. Zhdanov, Surf. Sci. 512 (2002) L331-L334.

[23] H. Li, X. Zhang, N. Liu, L. Ding, J. Tao, S. Wang, J. Su, L. Li, Y. Gao, Opt. Express 23 (2015) 21204-21212.

[24] Y. Zhang, Y. Liu, Z. L. Wang, Adv. Mater. 23 (2011) 3004-3013.

[25] L. Wen, K. M. Wong, Y. Fang, M. Wu, Y. Lei, J. Mater. Chem. 21 (2011) 7090.

[26] Z. Chen, B. Lai, J. Zhang, G. Wang, S. Chu, Nanotechnology 25 (2014) 295203.

[27] K. Sun, H. Zhang, L. Hu, D. Yu, S. Qiao, J. Sun, J. Bian, X. Chen, L. Zhang, Q. Fu, Z. Zhao, phys. status solidi (a) 207 (2010) 488-492.

[28] N. Liu, G. Fang, W. Zeng, H. Zhou, H. Long, X. Zou, Y. Liu, X. Zhao, Appl. Phys. Lett. 97 (2010) 243504.

[29] Z. Y. Zhang, C. H. Jin, X. L. Liang, Q. Chen, L. M. Peng, Appl. Phys. Lett. 88 (2006) 073102.

[30] C.-H. Lin, T.-T. Chen, Y.-F. Chen, Opt. Express 16 (2008) 16916-16922. 


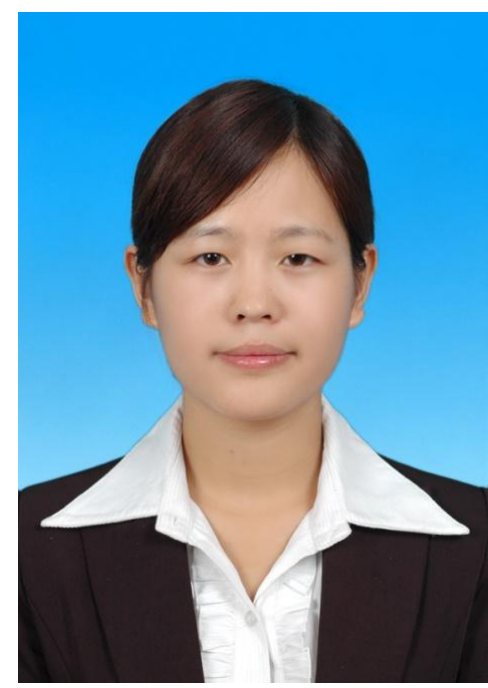

Haixia Li obtained her master (2012) from School of Physics in Huazhong University of Science and Technology (HUST), PR China. After that (2013), she joined the School of Physics as a Ph. D candidate- Her main research interest is semiconductor materials and piezo-optoelectronic devices.

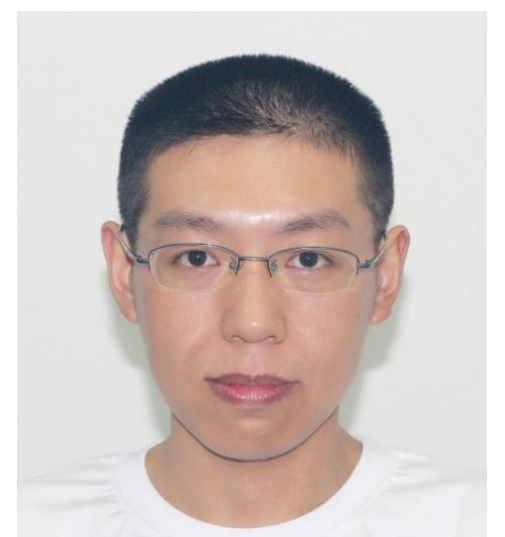

Dr. Nishuang Liu received his B.S. degree in Electronic Science and Technology (2006) and his Ph.D. in Microelectronics and Solid State Electronics (2011) from Wuhan University, PR China. Then, he joined School of Physics of HUST in 2011 as an Assistant Professor, and is now an Associate Professor. His main research interests include sensors, energy storage device and self-powered nanosystems. 


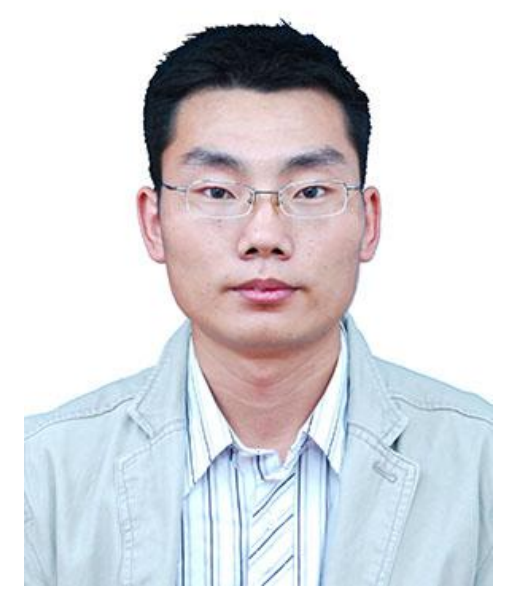

Dr. Xianghui Zhang obtained his bachelor (2007) and Ph.D. (2014) degree both form HUST, PR China. After that, he joined the Faculty of Physics and Electronic Science, Hubei University as an Assistant Professor. He is also a member of Hubei Collaborative Innovation Center for Advanced Organic Chemical Materials. His main research interest is lowdimensional semiconductor materials and optoelectronic devices.

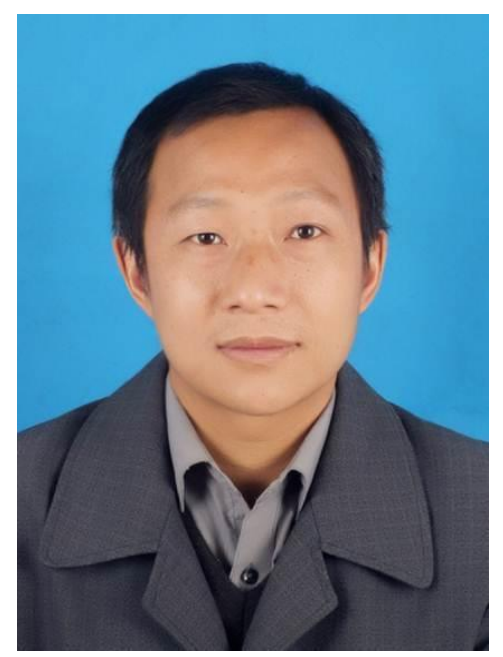

Dr. Jun Su is an engineer of electron microscope in Wuhan National Laboratory for Optoelectronics (WNLO), HUST. He received his Ph.D degree in Institute of Physics, Chinese Academy of Science in 2008. 


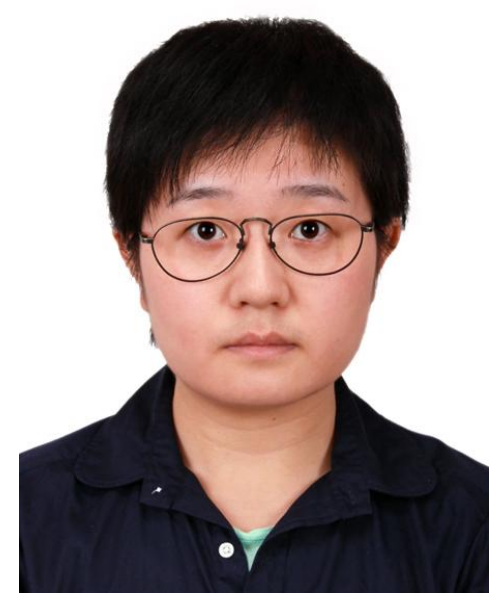

Dr. Luying Li received her Master degree in 2006 in physics department of Wuhan University, and PhD in 2011 in physics department of Arizona State University. In Dec, 2011, she joined WNLO, HUST. Her current research interest is about the relationship between atomic structures and specific physical properties of semiconductor nanomaterials.

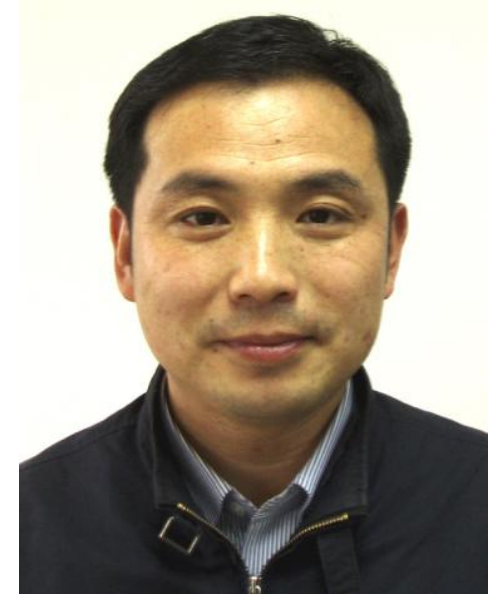

Dr. Yihua Gao received his PhD degree in Institute of Physics, Chinese Academy of Sciences (1998). Next he became a special researcher in the National Institute for Materials Science (NIMS) in Japan. In 2006, Dr. Gao accepted a professorship in HUST. His research interests include semiconductor film, microstructure, nanomaterials and nanodevices. 


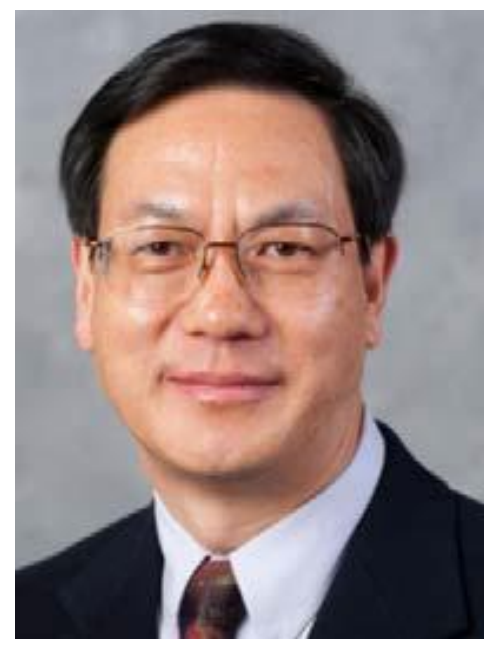

Dr. Zhonglin Wang is the Hightower Chair in Materials Science and Engineering, Regents' Professor, Engineering Distinguished Professor, and Director of the Center for Nanostructure Characterization at the Georgia Institute of Technology. His recent research focuses on the fields of nanogenerators, piezotronics, and piezo-phototronics for fabricating new electronic and optoelectronic devices. 
Graphical Abstract

Piezotronic and Piezo-phototronic Logic Computations Using Au

\section{Decorated ZnO Microwires}
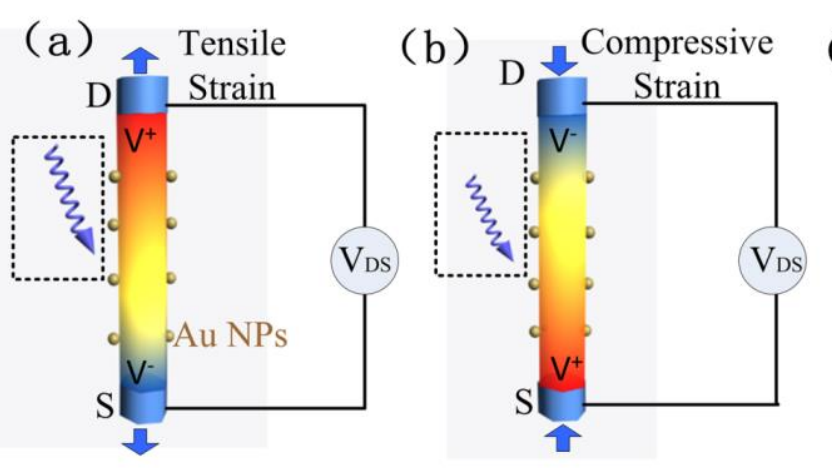

(c)
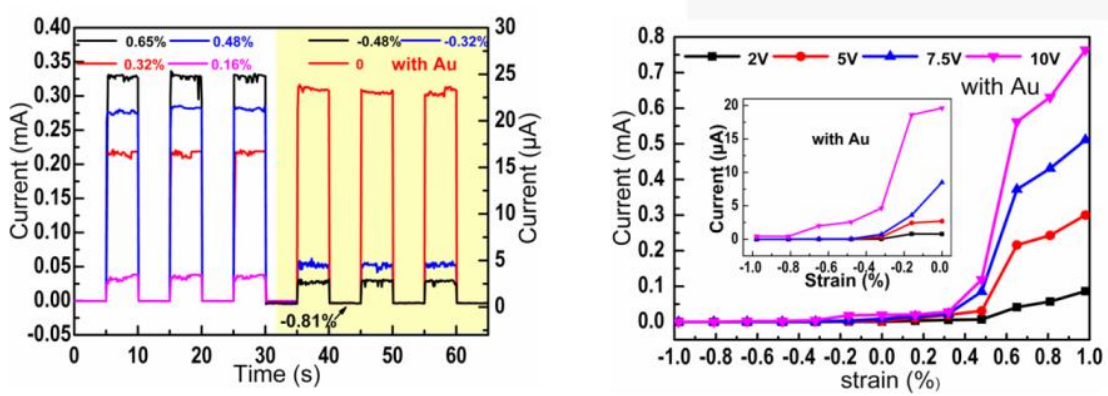